\title{
SIMULATION OF QUADCOPTER MOTION CONTROL IN VIRTUAL ENVIRONMENT SYSTEMS
}

\author{
M.V. MIKHAYLYUK ${ }^{1}$ AND E.V. STRASHNOV ${ }^{1}$ \\ ${ }^{1}$ Federal State Institution "Scientific Research Institute for System Analysis \\ of the Russian Academy of Sciences" (SRISA RAS), \\ Russia, 117218, Moscow, 36/1 Nakhimovskiy Avenue \\ e-mail:mix@niisi.ras.ru
}

\section{DOI: 10.20948/mathmon-2019-44-5}

Summary. The article considers the problem of quadcopter dynamics simulation and control in virtual environment systems. The proposed solution of this problem is based on the application of a mathematical model of quadcopter dynamics on the assumption that roll and pitch angles are small. In turn, the rotor dynamics is realized through a mathematical model of the electric motor based on its passport parameters. In this work, the feedback linearization method is used to control the quadcopter. With the application of this approach, the quadcopter's horizontal motion is achieved by the obtained expressions for the roll and pitch angles. The methods and approaches proposed in this article were implemented within the virtual environment complex and were tested on the example of a quadcopter model with control by a virtual remote controller.

\section{INTRODUCTION}

The quadcopter is an unmanned aerial vehicle with four rotors. Such devices are widely used for aerial photography and video, surveillance, search-and-rescue, and several other applications. The simulation of quadcopter motion control is an important and relevant area of research. This objective is required to find a such control strategy of quadcopter that allows to realize its vertical take-off and landing, rotations, movements in horizontal plane and attitude control when it performs various maneuvers and hovering at one point. Since the quadcopter's motion is described by nonlinear dynamic under-actuated system, then the design of quadcopter motion control is quite a challenge.

Currently, research is focused on the development of methods and algorithms for aerial vehicle control $[1,2]$. In particular, quadcopter control methods have been researched, including Lyapunov function [3-5], PID controllers [6-8] and linearization feedback [9-11]. The basis is the use of the Micro-Electro-Mechanical Systems (MEMS) sensors that consist of gyroscope, accelerometer, barometer and magnetometer, as well as data processing from ultrasonic sonar, LIDAR, GPS, etc. Since the readings of these sensors due to noise and the accumulation of integration errors are inaccurate, the issues of data refinement by constructing PID controllers, Kalman and low-pass filters are investigated in some works [6, $8,12]$.

A separate area of research is the use of virtual environment systems to implement quadcopter dynamic simulation and control. On the one hand, that allows to train the operator how to control a quadcopter using the remote controller, and on the other hand, the

2010 Mathematics Subject Classification: 70E60, 93B52, 97R60.

Key words and Phrases: Quadcopter, Simulation, Feedback Linearization Method, Remote Controller, Virtual Environment System. 
application of the virtual environment is convenient for approbation of the developed methods and algorithms of quadcopter control. Hence, the use of adequate mathematical models of the quadcopter dynamics and control for virtual environment systems is an important and current task. In this work, we consider a mathematical model of quadcopter dynamics in the form of Newton-Euler equations [13,14] on the assumption that the roll and pitch angles are small. These equations are supplemented by relationships for the rotor dynamics based on their passport parameters. To control the quadcopter, the feedback linearization method of the quadcopter's motion equations is applied. In this method, the quadcopter's horizontal motion is achieved by the obtained relationships for the roll and pitch angles. The methods and algorithms proposed in this article for quadcopter dynamic simulation and control were implemented within the virtual environment complex, developed in SRISA RAS. Their approbation in this software complex was carried out on the example of motion control of virtual quadcopter model (see the paper [15]) when operating by the virtual remote controller.

\section{QUADCOPTER MATHEMATICAL MODEL}

Consider a quadcopter with a design of the type X-configuration shown in Figure 1, in which the propellers are located at the ends of the frame rays. We define the following coordinate frames: the inertial frame $O X Y Z$ and the body frame $O_{B} X_{B} Y_{B} Z_{B}$, which moves along with a quadcopter.

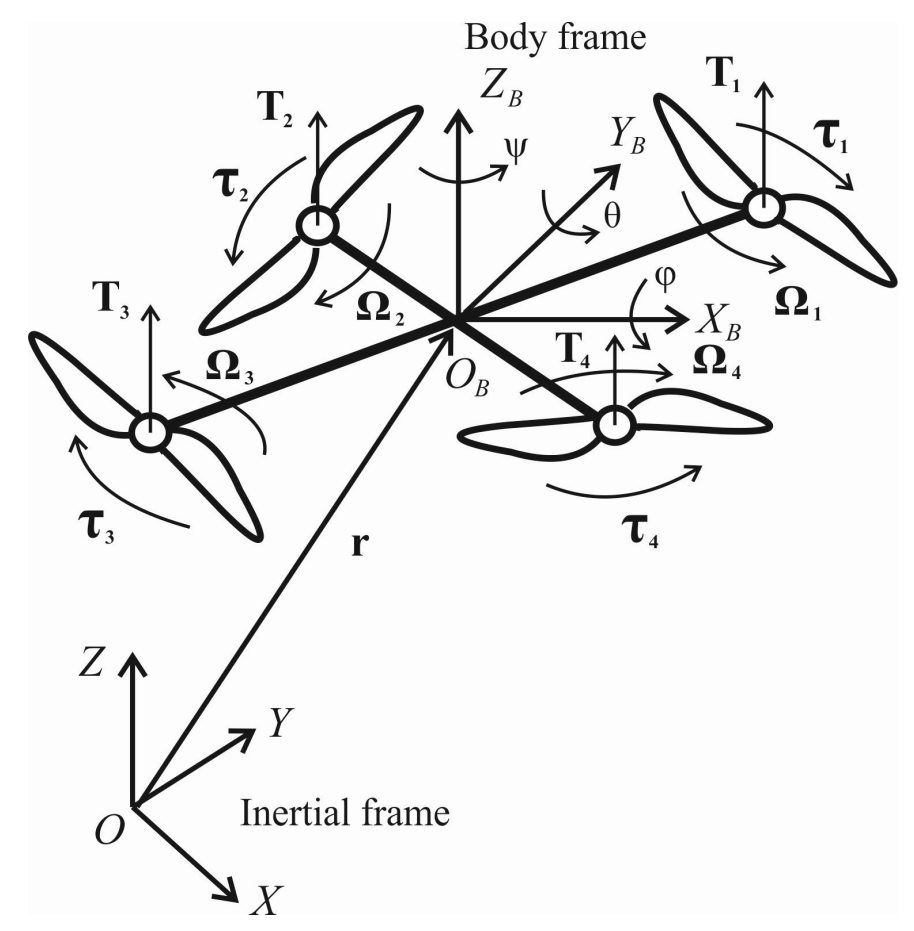

Figure 1. Schematic description of the quadcopter

The quadcopter's position is defined with radius vector $\mathbf{r}=\mathbf{O O}_{B}=(x, y, z)^{\mathrm{T}}$, and the attitude with $Z Y X$ Euler angles $\boldsymbol{\eta}=(\varphi, \theta, \psi)^{\mathrm{T}}$, where the rotations are first performed around $Z_{B}$-axis on the jaw angle $-\pi \leq \psi \leq \pi$, then around $Y_{B}$-axis on the roll angle 
$-\pi / 2<\theta<\pi / 2$ and finally around $X_{B}$-axis on the pitch angle $-\pi / 2<\varphi<\pi / 2$.

The transformation matrix $\mathbf{R}$ from the body frame to the inertial frame is given by

$$
\mathbf{R}=\left(\begin{array}{ccc}
c_{\psi} c_{\theta} & -s_{\psi} c_{\varphi}+c_{\psi} s_{\theta} s_{\varphi} & s_{\psi} s_{\varphi}+c_{\psi} s_{\theta} c_{\varphi} \\
s_{\psi} c_{\theta} & c_{\psi} c_{\varphi}+s_{\psi} s_{\theta} s_{\varphi} & -c_{\psi} s_{\varphi}+s_{\psi} s_{\theta} c_{\varphi} \\
-s_{\theta} & c_{\theta} s_{\varphi} & c_{\theta} c_{\varphi}
\end{array}\right),
$$

where notation $c_{\alpha}=\cos (\alpha)$ and $s_{\alpha}=\sin (\alpha)$ is used.

The quadcopters kinematics is defined by the linear velocity $\mathbf{v}=\left(v_{x}, v_{y}, v_{z}\right)^{\mathrm{T}}$ and the angular velocity $\boldsymbol{\omega}^{B}=\left(\omega_{x}^{B}, \omega_{y}^{B}, \omega_{z}^{B}\right)^{\mathrm{T}}$ that are given with respect to the inertial frame and the body frame, respectively. In this article, we assume that the angles $\varphi$ and $\theta$ during the simulation will be small. This leads (see the paper [8]) that the kinematic relations are following:

$$
\dot{\mathbf{r}}=\mathbf{v}, \dot{\boldsymbol{\eta}}=\omega^{B} .
$$

Consider all the forces and torques that are applied to the quadcopter. According to [12], rotations of rotors create thrusts $T_{i}=k \Omega_{i}^{2}, i=\overline{1,4}$ in the attachment points that are directed parallel to the axis $O_{B} Z_{B}$, where $\Omega_{i}$ and $k$ denotes, respectively, the angular velocity of rotor $i$ and the lift constant. We deal with the case when the quadcopter's center of mass is at the point $O_{B}$ (at origin of the body frame). Hence, the four rotors create total thrust $T=\sum_{i=1}^{4} T_{i}$ along the axis $O_{B} Z_{B}$, total torque $\tau_{\varphi}$ around the axis $O_{B} X_{B}$ and total torque $\tau_{\theta}$ around the axis $O_{B} Y_{B}$. Opposite the rotor rotation there are torques $\tau_{i}=b \Omega_{i}^{2}, i=\overline{1,4}$ around the axis $O_{B} Z_{B}$ due to the air drag, where $b$ denotes the drag constant of rotor. To compensate for the effect of this torques, the two rotors are spinning in the clockwise direction, while the two other in the counter-clockwise direction, which creates a total torque $\tau_{\psi}=\sum_{i=1}^{4}-\operatorname{sgn}\left(\Omega_{i}\right) \tau_{i}$ around the axis $O_{B} Z_{B}$. The expressions for the thrust $T$ and the torques $\tau_{\varphi}, \tau_{\theta}$ and $\tau_{\psi}$ can be represented as

$$
\begin{aligned}
& T=k\left(\Omega_{1}^{2}+\Omega_{2}^{2}+\Omega_{3}^{2}+\Omega_{4}^{2}\right), \\
& \tau_{\varphi}=k l_{y}\left(\Omega_{1}^{2}+\Omega_{2}^{2}-\Omega_{3}^{2}-\Omega_{4}^{2}\right), \\
& \tau_{\theta}=k l_{x}\left(-\Omega_{1}^{2}+\Omega_{2}^{2}+\Omega_{3}^{2}-\Omega_{4}^{2}\right), \\
& \tau_{\psi}=b\left(-\Omega_{1}^{2}+\Omega_{2}^{2}-\Omega_{3}^{2}+\Omega_{4}^{2}\right),
\end{aligned}
$$

where the values $l_{x}$ and $l_{y}$ are the rotor coordinate's positions in the body frame.

These forces and torques are combined in the force $\mathbf{f}_{s}$ and the torque $\boldsymbol{\tau}_{s}^{B}$ that are given with respect to the inertial frame and the body frame, respectively: 


$$
\mathbf{f}_{s}=\mathbf{R}\left(\begin{array}{c}
0 \\
0 \\
T
\end{array}\right), \quad \boldsymbol{\tau}_{s}^{B}=\left(\begin{array}{c}
\tau_{\varphi} \\
\tau_{\theta} \\
\tau_{\psi}
\end{array}\right) .
$$

Let's use the mathematical model [10] of air resistance, in which the drag force $\mathbf{f}_{d}$ depends on the square of the quadcopter's linear velocity:

$$
\mathbf{f}_{d}=-k_{d}|\mathbf{v}| \mathbf{v}
$$

where $k_{d}=c_{d} \frac{\rho S}{2}$ is the resistance coefficient, in which $c_{d}$ denotes the drag coefficient, $\rho$ the air density, $\mathrm{S}$ the cross-sectional area of the quadcopter to which covers air flow.

The quadcopter is assumed to be rigid body with the mass $m$ and inertia matrix $\mathbf{I}$. The rigid body dynamics is described as the Newton-Euler equations in the inertial frame:

$$
m \dot{\mathbf{v}}=m \mathbf{g}+\mathbf{f}, \mathbf{I} \dot{\boldsymbol{\omega}}=-\boldsymbol{\omega} \times \mathbf{I} \boldsymbol{\omega}+\boldsymbol{\tau},
$$

where $\mathbf{g}=(0,0,-g)^{\mathrm{T}}$ denotes the gravity acceleration, $\mathbf{f}$ the total force, $\boldsymbol{\tau}$ the total torque, $\mathbf{v}$ and $\boldsymbol{\omega}$ the quadcopter's linear and angular velocities.

Let's write the second equation (6) of the rotational motion in the body frame. In this case, the inertia matrix $\mathbf{I}$ is a constant diagonal matrix given by

$$
\mathbf{I}^{B}=\left(\begin{array}{ccc}
I_{x}^{B} & 0 & 0 \\
0 & I_{y}^{B} & 0 \\
0 & 0 & I_{z}^{B}
\end{array}\right) .
$$

Then, with the use Equations (1), (2), (4) and (5), Eq. (6) become

$$
\begin{aligned}
& m \ddot{x}=\left(s_{\psi} s_{\varphi}+c_{\psi} s_{\theta} c_{\varphi}\right) T-k_{d}|\dot{\mathbf{r}}| \dot{x}, \\
& m \ddot{y}=\left(-c_{\psi} s_{\varphi}+s_{\psi} s_{\theta} c_{\varphi}\right) T-k_{d}|\dot{\mathbf{r}}| \dot{y}, \\
& m \ddot{z}=-m g+c_{\theta} c_{\varphi} T-k_{d}|\dot{\mathbf{r}}| \dot{z}, \\
& I_{x}^{B} \ddot{\varphi}=\left(I_{y}^{B}-I_{z}^{B}\right) \dot{\theta} \dot{\psi}+\tau_{\varphi}, \\
& I_{y}^{B} \ddot{\varphi}=\left(I_{z}^{B}-I_{x}^{B}\right) \dot{\varphi} \dot{\psi}+\tau_{\theta}, \\
& I_{z}^{B} \ddot{\psi}=\left(I_{x}^{B}-I_{y}^{B}\right) \dot{\varphi} \dot{\theta}+\tau_{\psi},
\end{aligned}
$$

where the thrust $T$ and the torques $\tau_{\varphi}, \tau_{\theta}$ and $\tau_{\psi}$ are given by formulas (3).

The mathematical model (7) of the quadcopter's motion is supplemented by the rotor dynamics equations [16] based on their passport parameters:

$$
I_{m} \dot{\Omega}_{i}=M_{s}\left(U_{i}-\frac{\Omega_{i}}{\Omega_{\text {idle }}}\right), i=\overline{1,4},
$$


where $U_{i}$ denotes the voltage supplied to armature of the motor, $M_{s}$ the motor starting torque, $\Omega_{\text {idle }}$ the motor idling speed, $I_{m}=\frac{M_{s} t_{c}}{\Omega_{\text {idle }}}$ the motor inertia, in which $t_{c}$ is motor time constant.

Equations (7) and (8) define a mathematical model of quadcopter and rotor dynamics, where $T, \tau_{\varphi}, \tau_{\theta}$ and $\tau_{\psi}$ are given by formulas (3).

\section{QUADCOPTER CONTROL}

The quadcopter is an under-actuated mechanical system, since the number of actuators (four rotors) less than the number of degrees of freedom (three independent coordinates for the translational motion and three ones for the rotation). This means that we can control only four degrees of a quadcopter freedom at the same time. For example, the quadcopter's vertical motion (vertical take-off and landing) and attitude control are realized by changing of the thrust $T$ and the torques $\tau_{\varphi}, \tau_{\theta}$ and $\tau_{\psi}$. Next, we'll show how by changing the pitch $\varphi^{*}$ and the roll $\theta^{*}$ angles, it's possible to ensure the quadcopter's horizontal motion.

Formulas (3) are a system of linear equations with unknown rotor angular velocities $\Omega_{i}$, $i=\overline{1,4}$. For given $T^{*}, \tau_{\varphi}^{*}, \tau_{\theta}^{*}$ and $\tau_{\psi}^{*}$, in consideration of the rotor rotation directions (see the Figure 1), the solution of this system can be derived as

$$
\begin{aligned}
& \Omega_{1}^{*}=\sqrt{\frac{T^{*}}{4 k}+\frac{\tau_{\varphi}^{*}}{4 k l_{y}}-\frac{\tau_{\theta}^{*}}{4 k l_{x}}-\frac{\tau_{\psi}^{*}}{4 b}}, \Omega_{2}^{*}=-\sqrt{\frac{T^{*}}{4 k}+\frac{\tau_{\varphi}^{*}}{4 k l_{y}}+\frac{\tau_{\theta}^{*}}{4 k l_{x}}+\frac{\tau_{\psi}^{*}}{4 b}}, \\
& \Omega_{3}^{*}=\sqrt{\frac{T^{*}}{4 k}-\frac{\tau_{\varphi}^{*}}{4 k l_{y}}+\frac{\tau_{\theta}^{*}}{4 k l_{x}}-\frac{\tau_{\psi}^{*}}{4 b}}, \Omega_{4}^{*}=-\sqrt{\frac{T^{*}}{4 k}-\frac{\tau_{\varphi}^{*}}{4 k l_{y}}-\frac{\tau_{\theta}^{*}}{4 k l_{x}}+\frac{\tau_{\psi}^{*}}{4 b}} .
\end{aligned}
$$

In this article, we apply the feedback linearization method [17]. The main idea of this method is that the control variables are selected to algebraically transform nonlinear system (7) into linear ones, and then apply to it linear control techniques. The control variables for quadcopter are the thrust $T^{*}$ and the torques $\tau_{\varphi}^{*}, \tau_{\theta}^{*}$ and $\tau_{\psi}^{*}$. Substituting them into Eq. (9) we can obtain the required rotor velocities $\Omega_{i}^{*}, i=\overline{1,4}$. These velocities are ensured by computing the voltages $U_{i}, i=\overline{1,4}$ that transform the rotor dynamics in formula (8) to linear equations. Next, we consider the tasks of controlling the quadcopter's vertical and horizontal motion, its orientation and rotor rotations.

\subsection{Vertical motion control}

The quadcopter's vertical motion control is that to ensure changing of the coordinate $z$ as desired $z_{d}(t)$ with the velocity $\dot{z}_{d}(t)$. To do this we apply the feedback linearization method and transform the third equation (7) to the form

$$
m \ddot{z}=-m g+c_{\theta} c_{\varphi} T-k_{d}|\dot{\mathbf{r}}| \dot{z}=m\left(-k_{1, z}\left(z-z_{d}\right)-k_{2, z}\left(\dot{z}-\dot{z}_{d}\right)\right),
$$


where $k_{1, z}$ and $k_{2, z}$ are the linearization coefficients.

Selecting the thrust from this equation as

$$
T^{*}=\frac{1}{c_{\theta} c_{\varphi}}\left(m\left(g+k_{1, z}\left(z_{d}-z\right)+k_{2, z}\left(\dot{z}_{d}-\dot{z}\right)\right)+k_{d}|\dot{\mathbf{r}}| \dot{z}\right)
$$

we obtain the equation for residual $e_{z}(t)=z(t)-z_{d}(t)$ :

$$
\ddot{e}_{z}+k_{2, z} \dot{e}_{z}+k_{1, z} e_{z}=0 \text {. }
$$

Coefficients $k_{1, z}>0$ and $k_{2, z}>0$ can be selected so that the roots of the characteristic equation $\lambda^{2}+k_{2, z} \lambda+k_{1, z}=0$ will be different and have negative real part (in the case of complex roots). Then the solution $e_{z}(t)$ of this differential equation tends to zero as $t \rightarrow \infty$. This means that the coordinate $z$ of the quadcopter position converges to $z_{d}(t)$ with the velocity $\dot{z}_{d}(t)$.

\subsection{Attitude control}

The quadcopter attitude control is that the angles $\varphi, \theta$ and $\psi$ must be converge to desired values $\varphi_{d}(t), \theta_{d}(t)$ and $\psi_{d}(t)$ with their velocities $\dot{\varphi}_{d}(t), \dot{\theta}_{d}(t)$ and $\dot{\psi}_{d}(t)$. Let's transform the last three equations (7) using the feedback linearization method. Then the expressions for torques are selected as

$$
\begin{aligned}
& \tau_{\varphi}^{*}=\left(I_{z}^{B}-I_{y}^{B}\right) \dot{\theta} \dot{\psi}+I_{x}^{B}\left(k_{1, \varphi}\left(\varphi_{d}-\varphi\right)+k_{2, \varphi}\left(\dot{\varphi}_{d}-\dot{\varphi}\right)\right), \\
& \tau_{\theta}^{*}=\left(I_{x}^{B}-I_{z}^{B}\right) \dot{\varphi} \dot{\psi}+I_{y}^{B}\left(k_{1, \theta}\left(\theta_{d}-\theta\right)+k_{2, \theta}\left(\dot{\theta}_{d}-\dot{\theta}\right)\right), \\
& \tau_{\psi}^{*}=\left(I_{y}^{B}-I_{x}^{B}\right) \dot{\varphi} \dot{\theta}+I_{z}^{B}\left(k_{1, \psi}\left(\psi_{d}-\psi\right)+k_{2, \psi}\left(\dot{\psi}_{d}-\dot{\psi}\right)\right),
\end{aligned}
$$

where if we select the linearization coefficients as $k_{i, \varphi}>0, k_{i, \theta}>0$ and $k_{i, \psi}>0, i=1,2$, then the residuals $e_{\varphi}(t)=\varphi(t)-\varphi_{d}(t), e_{\theta}(t)=\theta(t)-\theta_{d}(t), e_{\psi}(t)=\psi(t)-\psi_{d}(t)$ tend to zero as $t \rightarrow \infty$. This means that the quadcopter's orientation angles converge to given functions $\varphi_{d}(t), \theta_{d}(t)$ and $\psi_{d}(t)$ with the velocities $\dot{\varphi}_{d}(t), \dot{\theta}_{d}(t)$ and $\dot{\psi}_{d}(t)$.

Substituting Equations (10) and (11) into Eq. (9), we'll get the required rotor velocities $\Omega_{i}^{*}, i=\overline{1,4}$, that let us to control the quadcopter's vertical motion and its orientation at the same time.

\subsection{Horizontal motion control}

The quadcopter horizontal motion control is that the position's coordinates $x$ and $y$ must be converge to desired values $x_{d}(t)$ and $y_{d}(t)$ with the velocities $\dot{x}_{d}(t), \dot{y}_{d}(t)$. We solve this problem by determining the angles $\varphi^{*}(t)$ and $\theta^{*}(t)$ of the quadcopter orientation and applying the feedback linearization method. For this, define the acceleration vector as 
$\mathbf{a}=\ddot{\mathbf{r}}=(\ddot{x}, \ddot{y}, \ddot{z})^{\mathrm{T}}$. Dividing the first three equations (7) by the mass $m$ yields

$$
\mathbf{a}=\mathbf{g}+\frac{1}{m} \mathbf{R}\left(\begin{array}{c}
0 \\
0 \\
T
\end{array}\right)-\tilde{k}_{d}|\dot{\mathbf{r}}| \dot{\mathbf{r}}, \tilde{k}_{d}=\frac{1}{m} k_{d} .
$$

Multiply the rotation matrix $\mathbf{R}^{\mathrm{T}}$ on both sides of the preceding equation, we obtain after transformations

$$
\left(\begin{array}{ccc}
c_{\psi} c_{\theta} & s_{\psi} c_{\theta} & -s_{\theta} \\
-s_{\psi} c_{\varphi}+c_{\psi} s_{\theta} s_{\varphi} & c_{\psi} c_{\varphi}+s_{\psi} s_{\theta} s_{\varphi} & c_{\theta} s_{\varphi} \\
s_{\psi} s_{\varphi}+c_{\psi} s_{\theta} c_{\varphi} & -c_{\psi} s_{\varphi}+s_{\psi} s_{\theta} c_{\varphi} & c_{\theta} c_{\varphi}
\end{array}\right)\left(\begin{array}{c}
a_{1}+\tilde{k}_{d}|\dot{\mathbf{r}}| \dot{x} \\
a_{2}+\tilde{k}_{d}|\dot{\mathbf{r}}| \dot{y} \\
a_{3}+g+\tilde{k}_{d}|\dot{\mathbf{r}}| \dot{z}
\end{array}\right)=\frac{1}{m}\left(\begin{array}{c}
0 \\
0 \\
T
\end{array}\right) .
$$

The first equation (12) is

$$
\left(\left(a_{1}+\tilde{k}_{d}|\dot{\mathbf{r}}| \dot{x}\right) c_{\psi}+\left(a_{2}+\tilde{k}_{d}|\dot{\mathbf{r}}| \dot{y}\right) s_{\psi}\right) c_{\theta}-\left(a_{3}+g+\tilde{k}_{d}|\dot{\mathbf{r}}| \dot{z}\right) s_{\theta}=0 .
$$

From here, the roll angle $\theta$ can be computed as

$$
\theta=\arctan \left(\frac{\left(a_{1}+\tilde{k}_{d}|\dot{\mathbf{r}}| \dot{x}\right) c_{\psi}+\left(a_{2}+\tilde{k}_{d}|\dot{\mathbf{r}}| \dot{y}\right) s_{\psi}}{a_{3}+g+\tilde{k}_{d}|\dot{\mathbf{r}}| \dot{z}}\right) .
$$

Consider the last two equations (12) and subtract the third equation multiplied by $s_{\varphi}$ from the second equation multiplied by $c_{\varphi}$. As a result, we get

$$
\frac{1}{m} T s_{\varphi}=\left(a_{1}+\tilde{k}_{d}|\dot{\mathbf{r}}| \dot{x}\right) s_{\psi}-\left(a_{2}+\tilde{k}_{d}|\dot{\mathbf{r}}| \dot{y}\right) c_{\psi} .
$$

To derive the expression for the pitch angle $\varphi$, first, it is necessary to determine the thrust $T$. For this, we introduce a vector $\mathbf{w}=\mathbf{R}^{T} \tilde{\mathbf{a}}$, in which

$$
\tilde{\mathbf{a}}=\left(a_{1}+\tilde{k}_{d}|\dot{\mathbf{r}}| \dot{x}, a_{2}+\tilde{k}_{d}|\dot{\mathbf{r}}| \dot{y}, a_{3}+g+\tilde{k}_{d}|\dot{\mathbf{r}}| \dot{z}\right)^{\mathrm{T}} \text {. }
$$

Then Eq. (12) can be represented as $\mathbf{w}=(0,0, T / m)^{\mathrm{T}}$. Since $\mathbf{R}$ is an orthogonal matrix (i.e., $\mathbf{R} \cdot \mathbf{R}^{\mathrm{T}}=\mathbf{I}$, where $\mathbf{I}$ is the identity matrix), then we obtain the following expression:

$$
|\mathbf{w}|^{2}=\left(\mathbf{R}^{\mathrm{T}} \tilde{\mathbf{a}}\right)^{\mathrm{T}} \mathbf{R}^{\mathrm{T}} \tilde{\mathbf{a}}=\tilde{\mathbf{a}}^{\mathrm{T}} \mathbf{R} \mathbf{R}^{\mathrm{T}} \tilde{\mathbf{a}}=|\tilde{\mathbf{a}}|^{2}=(T / m)^{2} .
$$

which is equivalent to

$$
T / m=|\tilde{\mathbf{a}}|=\sqrt{\left(a_{1}+\tilde{k}_{d}|\dot{\mathbf{r}}| \dot{x}\right)^{2}+\left(a_{2}+\tilde{k}_{d}|\dot{\mathbf{r}}| \dot{y}\right)^{2}+\left(a_{3}+g+\tilde{k}_{d}|\dot{\mathbf{r}}| \dot{z}\right)^{2}} .
$$

Combining this equation with Eq. (14) yields the pitch angle 


$$
\varphi=\arcsin \left(\frac{\left(a_{1}+\tilde{k}_{d}|\dot{\mathbf{r}}| \dot{x}\right) s_{\psi}-\left(a_{2}+\tilde{k}_{d}|\dot{\mathbf{r}}| \dot{y}\right) c_{\psi}}{\sqrt{\left(a_{1}+\tilde{k}_{d}|\dot{\mathbf{r}}| \dot{x}\right)^{2}+\left(a_{2}+\tilde{k}_{d}|\dot{\mathbf{r}}| \dot{y}\right)^{2}+\left(a_{3}+g+\tilde{k}_{d}|\dot{\mathbf{r}}| \dot{z}\right)^{2}}}\right) .
$$

Equations (13) and (15) for the roll and pitch angles are used to relate the rotational and translational motion of the quadcopter. Let's apply the feedback linearization method for the first three equations (7). Then, the expressions for the accelerations $a_{i}^{*}, i=\overline{1,3}$ are given by

$$
\begin{aligned}
& a_{1}^{*}=k_{1, x}\left(x_{d}-x\right)+k_{2, x}\left(\dot{x}_{d}-\dot{x}\right), \\
& a_{2}^{*}=k_{1, y}\left(y_{d}-y\right)+k_{2, y}\left(\dot{y}_{d}-\dot{y}\right), \\
& a_{3}^{*}=k_{1, z}\left(z_{d}-z\right)+k_{2, z}\left(\dot{z}_{d}-\dot{z}\right),
\end{aligned}
$$

where if we select the linearization coefficients as $k_{i, x}>0$ and $k_{i, y}>0, i=1,2$, then the residuals $e_{x}(t)=x(t)-x_{d}(t), e_{y}(t)=y(t)-y_{d}(t)$ tend to zero as $t \rightarrow \infty$. This means that the quadcopter's position coordinates converge to the given functions $x_{d}(t)$ and $y_{d}(t)$ with the velocities $\dot{x}_{d}(t), \dot{y}_{d}(t)$. Here, the expression for $a_{3}^{*}$ follows from the task of the quadcopter's vertical motion control.

Equations (16) for $a_{i}^{*}$ can be used to compute the desired angles $\varphi^{*}(t)$ and $\theta^{*}(t)$ from Equations (13) and (15). Then, the torques $\tau_{\varphi}^{*}$ and $\tau_{\theta}^{*}$ can be computed from Eq. (11) if we select $\varphi_{d}(t)=\varphi^{*}(t), \theta_{d}(t)=\theta^{*}(t), \dot{\varphi}_{d}(t)=0$ and $\dot{\theta}_{d}(t)=0$.

\subsection{Rotor rotations control}

This task is to compute the motor voltages $U_{i}, i=\overline{1,4}$ in order to ensure the required rotor angular velocities $\Omega_{i}^{*}$. Using the feedback linearization method for Equations (8) we get

$$
I_{m} \dot{\Omega}_{i}=M_{s}\left(U_{i}-\frac{\Omega_{i}}{\Omega_{\text {idle }}}\right)=-I_{m} k_{m, i}\left(\Omega_{i}-\Omega_{i}^{*}\right), i=\overline{1,4},
$$

where $k_{m, i}$ are the linearization coefficients.

In this case, we obtain the equations $\dot{e}_{i}+k_{m, i} e_{i}=0$ for the residuals $e_{i}=\Omega_{i}-\Omega_{i}^{*}$. If we select $k_{m, i}>0$, then the solutions $e_{i}(t)=C_{i} \exp \left(-k_{m, i} t\right), C_{i}=$ const of these equations tend to zero as $t \rightarrow \infty$.

Replacing $I_{m}=\frac{M_{s} t_{c}}{\Omega_{\text {idle }}}$ in Eq. (17) yields

$$
U_{i}=\frac{1}{\Omega_{\text {idle }}}\left(\left(1-t_{c} k_{m, i}\right) \Omega_{i}+t_{c} k_{m, i} \Omega_{i}^{*}\right), i=\overline{1,4} .
$$

Equations (18) for the voltages $U_{i}$ can be used to ensure the required rotor's angular 
velocities $\Omega_{i}^{*}$ of the quadcopter.

\section{SIMULATION RESULTS}

The proposed methods and approaches were implemented in the virtual environment system developed in SRISA RAS for virtual robot's simulation and control. This software package includes parts for control, dynamics simulation and visualization of virtual objects. The control system consists of the control panel editor, the block diagram editor and the module interface for signal processing that allows to implement the schemes of various complexity. Based on the actuator control signals, the updated coordinates and velocities of virtual objects (rigid bodies, electric motors, wheels, etc.) are obtained in the dynamics system. These coordinates (positions and orientations) are sent to the visualization system which provides high-quality visualization of the virtual scene in real time.

\begin{tabular}{|c|c|c|c|c|c|}
\hline Parameter & Value & Unit & Parameter & Value & Unit \\
\hline$m$ & 5 & $\mathrm{~kg}$ & $l_{x}$ & 0.2 & $\mathrm{~m}$ \\
\hline$g$ & 9.8 & $\mathrm{~m} / \mathrm{s}^{2}$ & $l_{y}$ & 0.25 & $\mathrm{~m}$ \\
\hline$I_{x}^{B}$ & 0.078 & $\mathrm{~kg} \mathrm{~m}^{2}$ & $k_{d}$ & 0.1536 & $\mathrm{~kg} / \mathrm{m}$ \\
\hline$I_{y}^{B}$ & 0.078 & $\mathrm{~kg} \mathrm{~m}^{2}$ & $M_{s}$ & 200 & $\mathrm{~N} \mathrm{~m}$ \\
\hline$I_{z}^{B}$ & 0.1367 & $\mathrm{~kg} \mathrm{~m}^{2}$ & $\Omega_{\text {idle }}$ & 3246 & $\mathrm{rpm}$ \\
\hline$k$ & $1.9851 * 10^{-4}$ & $\mathrm{~kg} \mathrm{~m}_{m}$ & $I_{m}$ & 0.0588 & $\mathrm{~kg} \mathrm{~m}^{2}$ \\
\hline$b$ & $7.436 * 10^{-6}$ & $\mathrm{~kg} \mathrm{~m}^{2}$ & $t_{c}$ & 0.1 & $\mathrm{~s}$ \\
\hline
\end{tabular}

Table 1. Parameter values for simulation

The feedback in this virtual environment complex is implemented using virtual sensors (see the paper [18]), such as position, attitude and angular rate sensors. The readings of these sensors are computed in the dynamics or visualization systems and then sent to the control system.

Parameter values for simulation of the quadcopter are presented in Table 1.

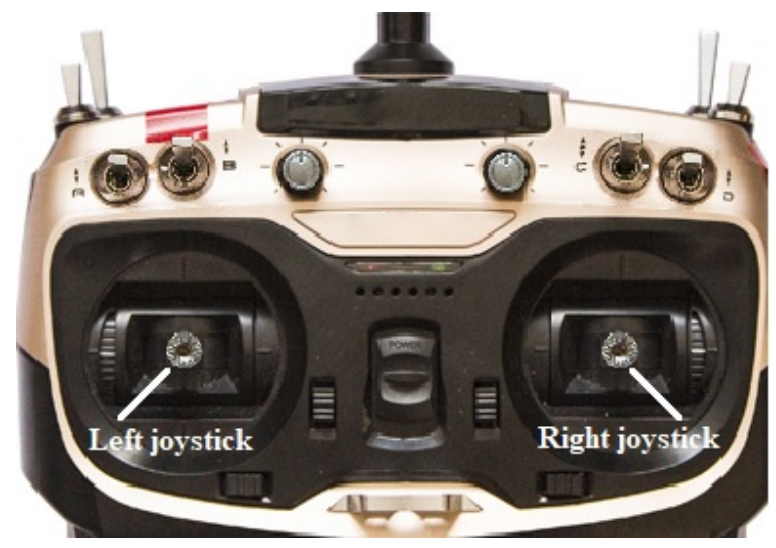

Figure 2. Quadcopter's virtual remote controller

The quadcopter simulation in the virtual environment system is implemented on the basis 
of the developed its virtual model and virtual remote controller (see the paper [15]) which is shown in Figure 2. Its motion is controlled by two joysticks. The horizontal and vertical positions of the left joystick specify the rate of the jaw angle $\dot{\psi}_{d}$ and the velocity $\dot{z}_{d}$, respectively. In the neutral position of the left joystick by selecting the desired values $z_{d}=$ const and $\dot{z}_{d}=0$ the quadcopter's height stabilization is realized. The right joystick displacements specify the desired roll $\theta_{d}$ and pitch $\varphi_{d}$ angles so that $\dot{\varphi}_{d}=\dot{\theta}_{d}=0$. In the neutral position of the right joystick by selecting the desired velocities $\dot{x}_{d}=\dot{y}_{d}=0$, the quadcopter's horizontal position stabilization is realized according to formulas (13) and (15) for the angles $\theta^{*}$ and $\varphi^{*}$. To ensure the condition of small angles, the limits $\left|\theta_{d}\right| \leq 30^{\circ}$ and $\left|\varphi_{d}\right| \leq 30^{\circ}$ are also imposed on the roll $\theta$ and pitch $\varphi$ angles.

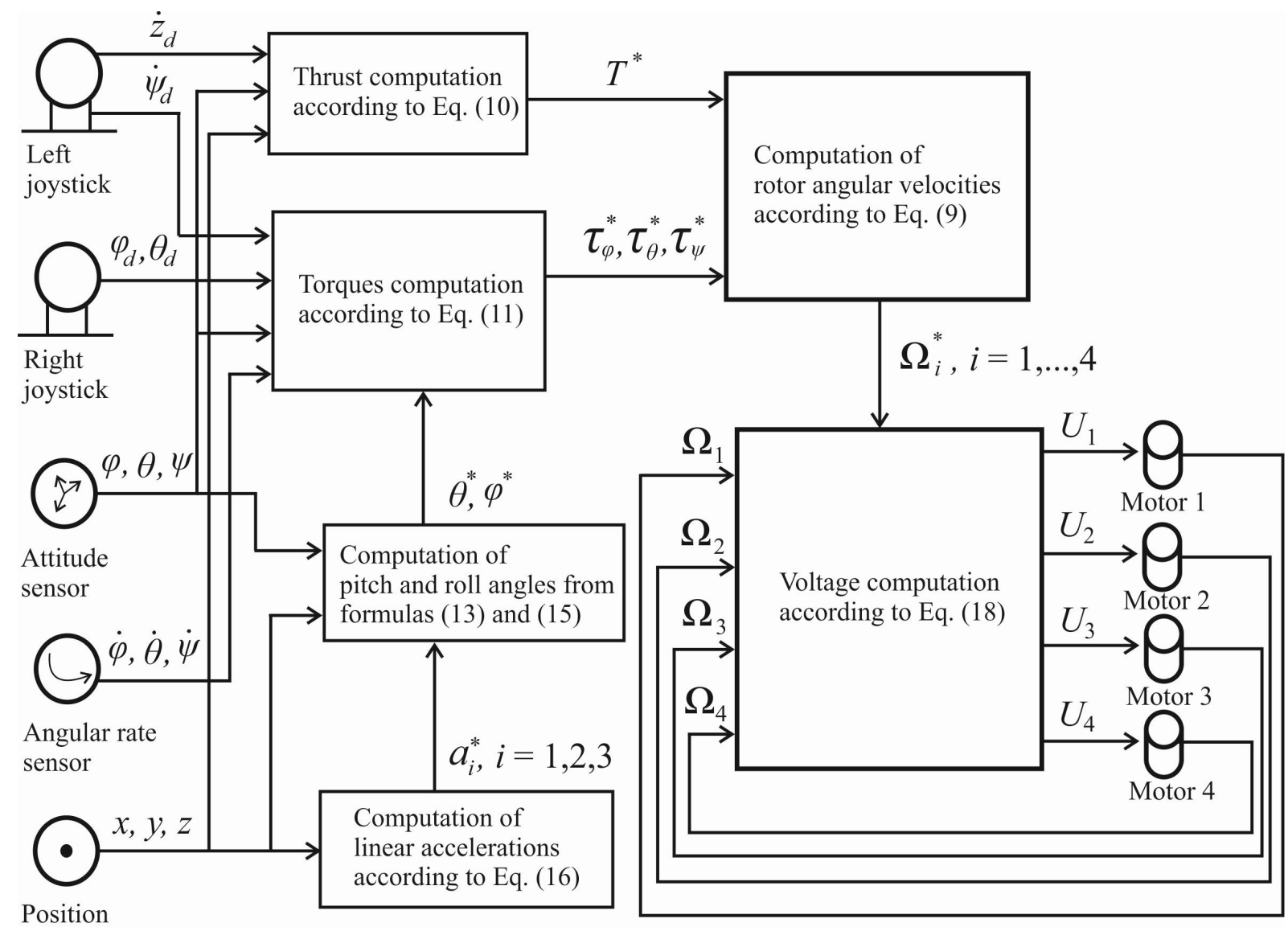

sensor

Figure 3. Structure of the quadcopter control scheme

The control algorithm of the virtual remote controller performs according to the block diagram scheme, the structure of which is shown in Figure 3. This scheme consists of control panel blocks, sensors, actuators (motors) and set of block libraries (arithmetic, trigonometric, logical, etc.). Each virtual sensor has its own block in the control scheme.

The position sensor computes the quadcopter's position coordinates and the linear velocities by differentiation of the receiving values. In its turn, the attitude sensor determines 
the quadcopter's Euler angles, while the angular rate sensor computes their derivatives. Also motors have the ability to compute the own rate of rotation. The joystick position changes and the sensor readings are sent to parts of the control scheme in which the computation of thrust, torques and rotor voltages according to formulas (10), (11), (16) and (18) is performed.

\begin{tabular}{|c|c|c|c|c|c|}
\hline Parameter & Value & Parameter & Value & Parameter & Value \\
\hline$k_{1, x}$ & 0.05 & $k_{1, y}$ & 0.05 & $k_{l, z}$ & 2 \\
\hline$k_{2, x}$ & 0.75 & $k_{2, y}$ & 0.75 & $k_{2, z}$ & 10 \\
\hline$k_{1, \varphi}$ & 12 & $k_{1, \theta}$ & 12 & $k_{1, \psi}$ & 1 \\
\hline$k_{2, \varphi}$ & 4 & $k_{2, \theta}$ & 4 & $k_{2, \psi}$ & 5 \\
\hline$k_{m, i}, i=\overline{1,4}$ & 200 & \multicolumn{5}{l}{} \\
\hline
\end{tabular}

Table 2. Parameters for the feedback linearization method

The linearization coefficients presented in Table 2 are selected so that to ensure the monotonic transient response, the absence of overshoot, the control performance and the nonnegativity of the radicals in Equations (9). Furthermore, an approximation $\sin (x) \approx x$ and $\tan (x) \approx x$ for small angles $x$ is used to decrease computations in the control scheme. The computed motor voltages are sent to the dynamic system, in which the quadcopter simulation using Equations (7) and (8) is implemented.

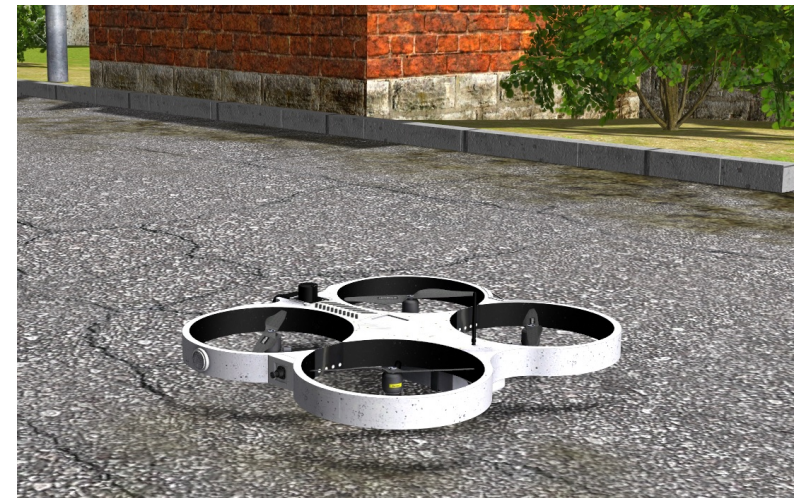

a)

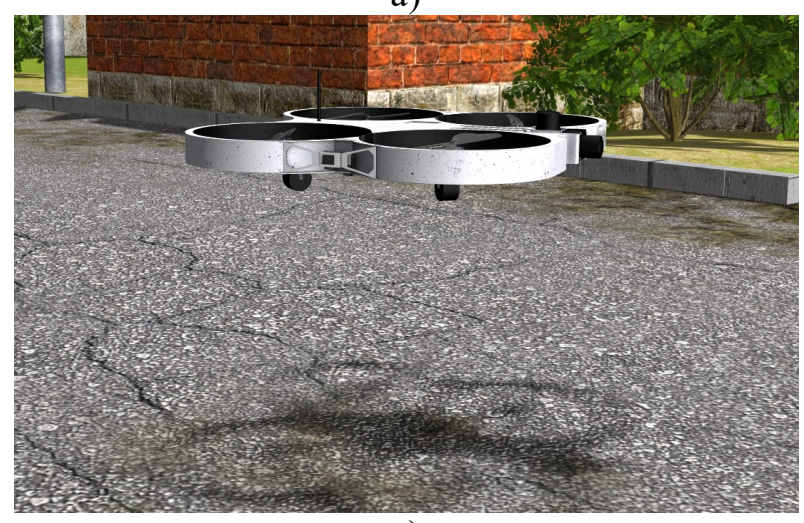

c)

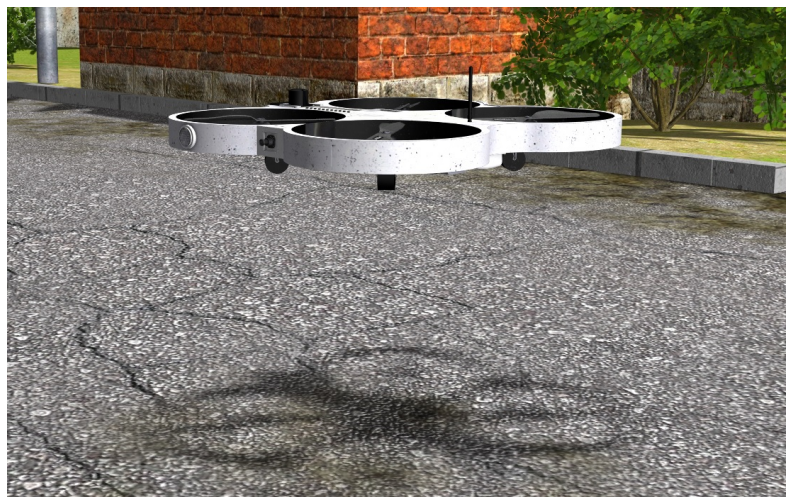

b)

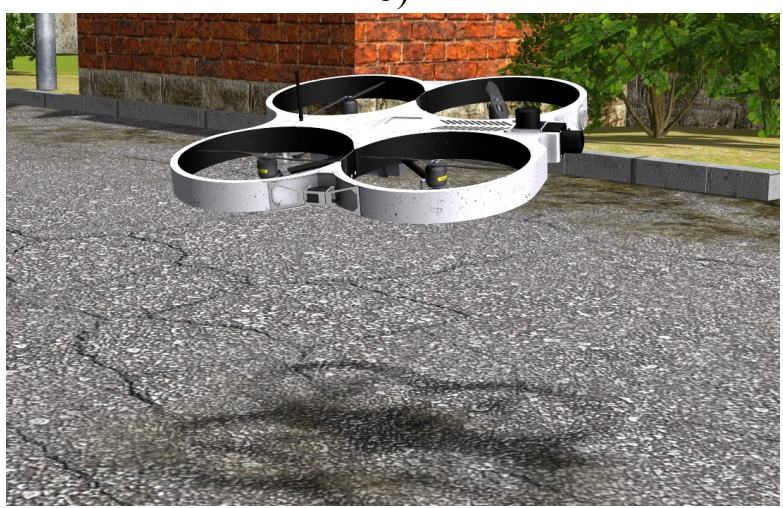

d)

Figure 4. Simulation of quadcopter motion control

Figure 4 shows the quadcopter's motion simulation in the virtual environment complex 
with using the remote controller. Four quadcopter's positions are shown: a) initial, b) at a height of $z_{d}=1$ meter, c) after rotating on the jaw angle $\psi_{d}=-135^{\circ}$, d) after rotating on the pitch angle $\varphi_{d}=30^{\circ}$ to start moving in the horizontal plane. The results show that the proposed methods and approaches for quadcopter control make it possible to realize horizontal and vertical motion, rotations, height and attitude stabilization.

\section{CONCLUSIONS}

This paper studied the problem of quadcopter motion control in virtual environment systems. To solve this problem, the feedback linearization method for the differential equations of quadcopter and actuator dynamics was used. In this case, when deriving the equations, it was assumed that during the simulation the roll and pitch angles are small. Approbation on the virtual quadcopter and remote controller models was showed that proposed methods and approaches for quadcopter control allow to realize its motion and stabilization. In the future, it is planned to develop methods and approaches for solving the problems of trajectory motion with any roll and pitch angles, modeling of real sensors with applying various filters for them.

Acknowledgements: The publication was made within the state task on carrying out basic scientific research (GP 14) on the topic (project) "34.9. Virtual environment systems: technologies, methods and algorithms of mathematical modeling and visualization" (00652019-0012).

\section{REFERENCES}

[1] G.K. Borovin, and V.V. Lapshin, "About a motion of free-floating space robot", Mathematica Montisnigri, 39, 67-78 (2017).

[2] G.K. Borovin, and V.V. Lapshin, "Motion control of a space robot", Mathematica Montisnigri, 41, 166-173 (2018).

[3] P. Castillo, A. Dzul, and R. Lozano, "Real-time stabilization and tracking of a four rotor minirotorcraft", IEEE Transactions on Control Systems Technology, 12 (4) (July 2004).

[4] I.C. Dickmen, A. Arisoy, and H. Temeltas, "Attitude control of a quadrotor", $4^{\text {th }}$ International Conference on Recent Advances in Space Technologies, $722-727$ (2009).

[5] Z. Zuo, "Trajectory tracking control design with command-filtered compensation for a quadrotor", IET Control Theory Appl., 4 (11), 2343-2355 (2010).

[6] T. Luukkonen, "Modelling and control of quadcopter", Independent research project in applied mathematics, Espoo: Aalto University (2011).

[7] H. Huang, G.M. Hoffmann, S.L. Waslander, and C.J. Tomlin, "Aerodynamics and control of autonomous quadrotor helicopters in aggressive maneuvering", IEEE International Conference on Robotics and Automation, 3277-3282 (May 2009).

[8] R. Beard, "Quadrotor dynamics and control rev 0.1", All Faculty Publications, No. 1325 (2008). Available at: https://scholarsarchive.byu.edu/facpub/1325/ [Accessed 7th March, 2019].

[9] F. Sabatino, Quadrotor control: modelling, nonlinear control design, and simulation, Master's Degree Project, Stockholm, Sweden (2015).

[10] A.E. Gur'ianov, Modelirovanie upravleniya kvadrokopterom, Engineering Bulletin of the Bauman MSTU, 8, 522 - 534 (2014).

[11] T.V. Glazkov, and A.E. Golubev, Otslezhivanie programmnogo izmeneniya uglovogo polozheniya kvadrokoptera, Mathematics and mathematical modeling, 5, 15-28 (2017).

[12] A. Gibiansky, 2012. Quadcopter dynamics and simulation (blog). Available at: 
http://andrew.gibiansky.com/blog/physics/quadcopter-dynamics/ [Accessed 7th March, 2019].

[13] M.V. Mikhaylyuk, E.V. Strashnov, and P.Yu. Timokhin, "Algorithms of multibody dynamics simulation using articulated-body method", Mathematica Montisnigri, 39, 133-143 (2017).

[14] M.V. Mikhaylyuk, and E.V. Strashnov, "Simulation of articulated rigid bodies with penalty method", Mathematica Montisnigri, 27, 91-106 (2013).

[15] E.V. Strashnov, L.A. Finagin, and I.N. Mironenko, Tekhnologiya sozdaniya virtual'nyh modelej kvadrokoptera i pul'ta upravleniya, Proceedings of SRISA RAS, 9 (1), 49-55 (2019).

[16] E.V. Strashnov, and M.A. Torgashev, Modelirovanie dinamiki ehlektroprivodov virtual'nyh robotov $v$ imitacionno-trenazhernyh kompleksah, Mekhatronika, Avtomatizatsiya, Upravlenie, 17 (11), 762-768 (2016).

[17] A.P. Krishchenko, Stabilizaciya programmnyh dvizhenij nelinejnyh sistem, Engineering Cybernetics, 6, 103-112 (1985).

[18] M.V. Mihajlyuk, E.V. Strashnov, and D.M. Loginov, Modelirovanie datchikov v sistemah virtual'nogo okruzheniya, Proceedings of SRISA RAS, 8 (2), 70-76 (2018).

Received January 28, 2019 